\title{
ORIGEN Y EVOLUCIÓN DE LA GIMNASIA RÍTMICA MASCULINA: UN CAMINO HACIA LA IGUALDAD DE DERECHOS $^{1}$
}

\author{
Carmen Cambiella Pereira \\ Universidad Autónoma de Madrid \\ carcamper7@gmail.com \\ $M^{\mathbf{a}}$ Eugenia Martínez Gorroño \\ Universidad Autónoma de Madrid \\ eugenia.martinez@uam.es
}

Fecha de recepción: septiembre 2020

Fecha de aceptación: noviembre 2020

http://doi.org/10.15366/citius2020.13.2.005

\section{Resumen:}

Los principios olímpicos recogidos en la Carta Olímpico reflejan la filosofía del Movimiento Olímpico. Uno de esos principios es la no discriminación por razones de sexo o de género. La gimnasia rítmica es uno de los pocos deportes olímpicos con participación exclusivamente femenina. El objetivo de esta investigación ha sido rescatar el origen de este deporte y su evolución, tanto a nivel nacional como internacional, y reflexionar si actualmente, y en relación con este deporte, se cumplen en la práctica los principios fundamentales base del ideario Olímpico. Con tal perspectiva, se realizó el análisis documental de la Carta Olímpica, de la Declaración Universal de Derechos Humanos y de la documentación de los archivos de la Real Federación Española de Gimnasia (RFEG), así como de fuentes hemerográficas especializadas. Por otro lado, se celebró una entrevista semiestructurada de final abierto al gimnasta español Rubén Orihuela Gavilán. La información y los resultados fueron objeto de contraste y triangulación, y mostraron que la gimnasia rítmica no tiene restricciones en cuanto a su práctica, en cambio a nivel competitivo, los eventos en los que los gimnastas pueden participar son escasos. En este sentido en España se documentan impulsos equilibradores de estas circunstancias, mostrando un avance pionero en este sentido, como la concesión de la primera licencia federativa masculina del mundo o la celebración del primer Campeonato Nacional Masculino de Gimnasia Rítmica celebrado en Gijón el 2009. Por todo ello, es preciso señalar que tanto la Federación Internacional de Gimnasia (FIG) como las federaciones nacionales tienen en sus manos la posibilidad de generar el impulso que la gimnasia rítmica masculina necesita, propiciando la visibilidad de este deporte y el aumento de su práctica, aspecto que también condiciona la participación olímpica.

Palabras clave: gimnasia rítmica masculina, Movimiento Olímpico, Derechos Humanos, igualdad.

Title: ORIGIN AND EVOLUTION OF MALE RHYTHMIC GYMNASTICS: A PATH TO EQUAL RIGHTS

\begin{abstract}
:
The Olympic principles included in the Olympic Charter reflect the philosophy of the Olympic Movement. One of these principles is non-discrimination based on sex or gender. Rhythmic gymnastics is one of the few Olympic sports with exclusively female participation. The objective of this research has been to rescue the facts that suppose its origin and evolution, both nationally and internationally, and reflect

\footnotetext{
${ }^{1}$ El contenido del presente artículo es la adaptación y la versión en español del trabajo que fue presentado para postular la candidatura de Carmen Cambiella Pereira al 27 Seminario International en Estudios Olímpicos para estudiantes post-graduados convocado por la Academia Olímpica Internacional. Después de haber conseguido con este trabajo la representación de la Academia Olímpica Española para Carmen Cambiella en el citado seminario, el contenido del trabajo de investigación fue presentado y defendido por ella en las sesiones del citado seminario celebrado este año de forma telemática, desde la sede de la Academia Olímpica Internacional en Olimpia (Grecia) en septiembre del 2020.
} 
on whether in the current situation, and in relation to this sport, the fundamental principles that are at the base of the Olympic are fulfilled in practice. Therefore, the documentary analysis of the Olympic Charter, the Universal Declaration of Human Rights and the documentation of the archives of the Royal Spanish Gymnastics Federation, as well as specialized newspaper sources, was carried out. On the other hand, a semi-structured interview was conducted with the Spanish gymnast Ruben Orihuela Gavilan. The information and results were contrasted and triangulated and showed that rhythmic gymnastics has no restrictions regarding its practice, however at a competitive level, the events in which gymnasts can participate are scarce. In this sense in Spain balancing impulses of this circumstances are documented, showing a pioneering advance on this matter, such as the granting of the first male license in the world, or the celebration of the first National Male Rhythmic Gymnastics Championship held in Gijon in 2009.Therefore, it should be noted that it is now in the hands of the International Gymnastics Federation and the national federations the possibility of generating the boost that male rhythmic gymnastics needs, promoting the visibility of this sport and the increase in its practice, aspects that also conditions Olympic participation.

Key words: rhythmic gymnastics men/male, Olympic Movement, Human Rights, equality.

\section{Introducción}

La gimnasia rítmica de competición es un deporte que engloba a gimnastas que, con licencia federativa participan en eventos deportivos organizados por las federaciones Internacionales y nacionales, y en el caso de España, también autonómicas.

Es una disciplina deportiva reconocida desde 1962 por la Federación Internacional de Gimnasia (FIG), y se incluyó en el Programa Olímpico desde la edición de los Juegos de Los Ángeles en 1984. La gimnasia rítmica ha sido tradicionalmente una disciplina deportiva practicada por mujeres. Sin embargo, algunas federaciones de ciertos países, como España, iniciaron hace algunos años la organización de competiciones de gimnasia rítmica masculina. Entendemos que, con ello, se ha pretendido dar un paso en la evolución del deporte, siguiendo, tanto las directrices de la Declaración Universal de Derechos Humanos, de la Asamblea General de la ONU, como de los Principios Fundamentales del ideario olímpico, recogidos en la Carta Olímpica. En ésta última, se afirma:

"La práctica deportiva es un derecho humano. Toda persona debe tener la posibilidad de practicar deporte sin discriminación de ningún tipo y dentro del espíritu olímpico, que exige comprensión mutua, espíritu de amistad, solidaridad y juego limpio" (Carta Olímpica. Principio Fundamental $\left.n^{\circ} 4\right)$.

Por otra parte, el Principio Fundamental número 6 de la misma Carta, establece:

"El disfrute de los derechos y libertades establecidos en esta Carta Olímpica debe garantizarse sin ningún tipo de discriminación, ya sea por raza, color, sexo, orientación sexual, idioma, religión, opiniones políticas o de otra índole, origen nacional o social, riqueza, nacimiento u otra condición” (Carta Olímpica. Principio Fundamental nº 6).

La FIG es la encargada, por una parte, de la organización de competiciones internacionales, y, por otra, de establecer la normativa de esta disciplina deportiva, siendo la gimnasia rítmica la única disciplina dentro de la FIG que solamente permite la participación de mujeres. Como consecuencia, en el programa olímpico, no hay participación masculina en competiciones de gimnasia rítmica.

\section{Objeto de estudio}

Partiendo de los principios olímpicos de igualdad ya identificados en la Carta Olímpica, nos planteamos el comienzo de este trabajo centrado en la gimnasia rítmica masculina de competición. Al ser el único deporte olímpico, junto con la natación sincronizada, con participación exclusivamente femenina, pretendemos rescatar los hechos que suponen su origen 
y evolución, tanto a nivel nacional como internacional, y reflexionar si en la situación actual, y en relación a este deporte, se cumplen en la práctica los principios fundamentales que están en la base del ideario Olímpico.

\section{Objetivos}

El objetivo general de esta investigación ha sido conocer la evolución de la participación masculina en las competiciones nacionales e internacionales de gimnasia rítmica, partiendo de los criterios de igualdad que se establecen en los Principios fundamentales del Olimpismo. Por tanto, los objetivos específicos de este trabajo son:

- Conocer el proceso del inicio de la gimnasia rítmica masculina en España, así como su evolución hasta la actualidad.

- Conocer la realidad actual de la gimnasia rítmica masculina a nivel internacional, así como su evolución en los diferentes países donde se practica y se compite en este deporte.

- Reflexionar sobre la realidad de la gimnasia rítmica, como deporte olímpico en relación con el cumplimiento de los principios fundamentales del Olimpismo y los Derechos Humanos, en términos de igualdad y no discriminación.

\section{Metodología}

El objeto de estudio de este trabajo supone la recuperación histórica de un fragmento de la Historia Contemporánea en la que hay fuentes vivas. Éstas, son personas que nos permiten reconstruir, gracias a su testimonio, unos aspectos históricos que difícilmente podrían conocerse a partir de otras fuentes. Por tanto, se trata de una investigación de lo que se ha denominado como "Historia del Tiempo Presente" (Bédarida, F. 1998), que nos condiciona a una metodología específica, dentro de la investigación de tipo histórico.

De acuerdo con Soto Gamboa, el investigador de la Historia del Tiempo Presente (HTP) "tiene la posibilidad de apreciar y observar las mentalidades, la percepción de los contemporáneos sobre el tema y sus propias vivencias" (Soto Gamboa, 2004, p.137).

Siguiendo los criterios establecidos en la preparación metodológica recibida en la Universidad Autónoma de Madrid, para enfrentar una investigación de la HTP, en primer lugar, procedidos al proceso de identificación y categorización de las posibles fuentes primarias. Y así, en el transcurso de la "aproximación al objeto de estudio", nuestra experiencia deportiva nos permitió obtener información de primera mano sobre las vivencias de varios deportistas de esta especialidad y sus circunstancias en relación con el cumplimiento de los Principios Olímpicos. Todo ello, fue definitivo para seleccionar y categorizar como fuente primaria al que se podría considerar pionero de la gimnasia rítmica en España, Rubén Orihuela Gavilán: 9 veces campeón nacional y primera licencia federativa masculina en el mundo en gimnasia rítmica. Se trata del primer gimnasta masculino en participar en un Campeonato Nacional de Gimnasia Rítmica en España.

Tras el contacto con esta definitiva fuente primaria, el mostró una gran predisposición para ofrecernos su testimonio. Así, se realizó una entrevista semiestructurada de final abierto al citado gimnasta, que debido a la situación actual de confinamiento por la pandemia del Covid-19 se realizó por videoconferencia. Se elaboró previamente un guion coherente con los objetivos ya establecidos, siguiendo los aspectos metodológicos propuestos por Hammer y Wildavsky (1990).

Por otra parte, igualmente hemos categorizado como fuentes primarias: la Carta Olímpica y de la Declaración Universal de Derechos Humanos, (Asamblea General de la ONU), la documentación que obra en los archivos de la Real Federación Española de Gimnasia (RFEG), 
relativa a nuestro objeto de estudio. Los dos primeros documentos contienen los principios ideológicos que pretendemos analizar e identificar en el fragmento de la historia deportiva que es objeto de estudio. Con respecto a la documentación de la Federación, fue utilizada aquella que contenía información relativa a la evolución histórica deportiva de esta especialidad en nuestro país. A todas las fuentes escritas se les ha aplicado la técnica del "Análisis Documental", que igualmente se empleó para extraer información y datos de fuentes hemerográficas especializadas, que contenían noticias específicas, que han sido definitivas en el proceso de contraste y triangulación para la reconstrucción histórica que nos hemos propuesto.

\section{Resultados}

\subsection{Origen, evolución y situación actual de la gimnasia rítmica masculina}

La gimnasia rítmica a lo largo de la historia ha tenido diferentes denominaciones: gimnasia moderna, gimnasia rítmica moderna, gimnasia rítmica deportiva...Es un deporte relativamente joven. Fue admitida en el Programa Olímpico en los Juegos Olímpicos (JJOO) de Los Ángeles 1984 (Mendizábal Albizu, 2000). Algunas personas clave en el desarrollo de este deporte fueron Isidora Duncan, Émile-Jaques Dalcroze y Rudolf Bode. En su inicio fue una práctica exclusiva para mujeres, y actualmente, aún sigue siendo una de las dos únicas disciplinas deportivas representada en los JJOO por un solo género: el femenino.

Ante una situación, que podría considerarse como una falta del cumplimiento de los Principios Olímpicos, la FIG creó lo que se conoce como gimnasia rítmica japonesa, que según afirmaba Rubén Orihuela, en la entrevista que con él mantuvimos “...fue creada por la FIG en un intento por mantener la gimnasia rítmica dentro de los JJOO, pero es una gimnasia diferente, más marcial" (Orihuela Gavilán R., 2020). Esta modalidad difiere de la gimnasia rítmica tradicionalmente practicada por mujeres. Orihuela afirmaba, que, a pesar de que está extendida por varios países, "... el hecho de que sea tan marcial es probable que sea lo que dificulte su expansión por occidente" (Ibídem).

Paralelamente a la práctica de la gimnasia rítmica japonesa, en otros lugares del mundo, algunos hombres comenzaron a practicar la gimnasia rítmica. Pero no se trataba de la versión japonesa, sino de "la gimnasia rítmica femenina, pero practicada por los hombres, con su misma normativa" (Ibídem). Este tipo de gimnasia es la que se practica en España, y un ejemplo de ello es precisamente, Rubén Orihuela, primer gimnasta masculino en obtener una licencia federativa de gimnasia rítmica. Así, él mismo confirmaba:

"Yo no fui el primer chico que practicó gimnasia rítmica, antes que yo hubo otros chicos que practicaban gimnasia en sus clubs, comunidades autónomas y países. Lo que si fui fue el primer gimnasta con licencia federativa en el mundo, es decir, la primera vez que se registró una licencia de un chico en gimnasia rítmica" (Ibídem).

Rubén Orihuela Gavilán es un gimnasta español de origen valenciano, que pudo participar por primera vez en una competición nacional en el año 2005, junto con otros dos gimnastas más, en categoría OPEN, categoría que incluía a hombres y mujeres. Tuvieron que esperar al 2007 para poder tener opción a obtener una medalla. En 2009, probablemente debido al aumento de la participación masculina, la Real Federación Española de Gimnasia (RFEG) procedió a llevar a cabo una consulta a la FIG sobre qué normativa debía aplicarse en las competiciones con participación de ambos géneros. La FIG contestó: "la gimnasia rítmica es un deporte sólo para mujeres y la FIG no tiene reglas para la competición de hombres" (Robles, 2018). Después la RFEG tomó la decisión de prohibir la participación masculina en el Campeonato Nacional de Gimnasia Rítmica, dejando en manos de las federaciones autonómicas la posibilidad o no de permitir participación masculina en competiciones de ámbito provincial. 
Ante esta decisión de la RFEG, Rubén recordaba los hechos que posteriormente se sucedieron. Él se puso en contacto con los medios de comunicación y dio a conocer la situación que se estaba viviendo. Aquella publicidad, "no puso en buen lugar a la federación. No les agradó. Eso me lo dijeron". Por otra parte- Almudena Cid Tostado, gimnasta española cuatro veces olímpica, inició contactos con el Ministerio de Igualdad. Producto de aquellas conversaciones la federación cambió de opinión. Permitió la participación masculina en un campeonato nacional, y se celebró por primera vez un Campeonato Nacional Masculino de Gimnasia Rítmica (Tapigym, 2018). Ello suponía un especial hito histórico para la gimnasia rítmica: un campeonato de España con participación y clasificación exclusivamente para hombres, que tuvo lugar en Gijón, el año 2009.

Desde entonces, y hasta nuestros días, se sigue celebrando anualmente el Campeonato Nacional Masculino en nuestro país, en el que además se permite la participación de gimnastas de otros países. Todo ello supone, no solo un hecho sin precedentes, que ha marcado la historia de esta especialidad a nivel nacional e internacional, sino un antecedente único que cumple el "valor educativo del buen ejemplo", como también consta en la Carta Olímpica. Por otra parte, la labor pionera de España ha seguido consolidándose en este sentido, ya que en los últimos años también se ha comenzado a celebrar la Copa de la Reina Masculina, otro campeonato nacional más en el que los hombres pueden participar.

La evolución positiva y pionera que sigue liderada por nuestro país se continúa consolidando a partir de otros impulsos. Así, este mismo año la RFEG ha creado la Primera Categoría Masculina, integrada por los 6 mejores gimnastas de España, cuyo listado ha sido publicado en junio de 2020. Este hecho fue resaltado por Rubén Orihuela quien afirmaba:

"Todos los chicos que ahora se encuentran en esta categoría van a entrenar como nunca han hecho para dar lo mejor de si'". "Con la primera categoría que se ha creado recientemente se abre un camino para que, aparte de crecer en cantidad, como se ha hecho en años anteriores, ahora crezcamos en calidad. Además, esto permite que los gimnastas senior tengan más opciones de conseguir medalla debido a que los que siempre quedaban en pódium, ahora han ascendido a Primera Categoría”. (Orihuela Gavilán R., 2020).

Por otra parte, es preciso mencionar algunos otros impulsos pioneros, que se han ido produciendo igualmente en nuestro país, en la búsqueda de la igualdad en esta especialidad deportiva. Hasta hace poco tiempo los campeonatos masculinos que se realizaban en España solo contemplaban la categoría individual; sin embargo, en el Euskalgym, un evento internacional de gimnasia que se celebra cada año en País Vasco, además de la categoría masculina individual, se permite la participación de conjuntos mixtos. Sobre ello afirmaba Orihuela:

"Euskalgym es un evento que da la posibilidad de mostrarse a los gimnastas en un entorno internacional y único. Seguramente no sea el único evento internacional, pero si probablemente el único que se realiza periódicamente cada año” (Ibídem).

Sumado a esto, en la reciente modificación de la normativa publicada por la RFEG en junio de 2020 se ha incluido la participación de conjuntos mixtos (open mixto), en todas las categorías de edades. Estos conjuntos pueden conformarse en cuatro modalidades: 4 chicas y 1 chico, 3 chicas y 1 chico, 2 chicas y 1 chico y 1 chica y 4 chicos (Normativa técnica GR, RFEG, junio 2020, p. 23). El próximo Campeonato de España de Conjuntos será el primero en el que, tanto chicos como chicas, puedan competir formando conjunto. En caso de que no haya un mínimo de 3 conjuntos mixtos de la misma categoría, estos conjuntos participarán en la misma categoría que los femeninos, habiendo dos clasificaciones: la "tradicional", que solo incluye conjuntos femeninos, y la open-mixta, que incluye los conjuntos femeninos y los mixtos. La propia RFEG en su normativa técnica publicada en junio de 2020 afirma que: "Durante los años 2020 y 2021, se realizará esta competición con carácter experimental y según los resultados y participación, se estudiará su continuidad o no”. (Normativa técnica GR, RFEG, junio 2020, p. 28). 
A pesar de todos los citados impulsos promovidos en los últimos años, la gimnasia rítmica masculina aún dista de ser olímpica. En primer lugar, porque la única reconocida por la FIG es la japonesa, y, en segundo lugar, porque para participar en los Juegos se necesita un mínimo de participación de países en los cuáles esta modalidad sea oficial. Por ello, el camino hacia los JJOO parece estar en manos de la FIG y de las federaciones nacionales de gimnasia de los diferentes países donde hay practica y competiciones masculinas. Ellos son, además de España: México, Argentina, Brasil, Italia, Grecia y Francia.

Varios proyectos han sido emprendidos para el año próximo que pueden seguir impulsando la presencia olímpica. En 2021, en Brasil se prevé oficializar la gimnasia rítmica masculina. En el campeonato nacional de Francia, se ha dispuesto que la nota final del equipo para la clasificación general, salga de la suma de la nota de un ejercicio de conjunto y de dos ejercicios individuales, y estos dos últimos a su vez pueden ser realizados, por chicos o chicas, tanto franceses como de otras nacionalidades. Estos aspectos, hacían reflexionar a Rubén Orihuela en la entrevista que con él mantuvimos, que quizá no haya que esperar mucho para poder verlos disfrutar de unos Juegos.

\subsection{La gimnasia rítmica: experiencia de vida de Rubén Orihuela Gavilán}

Rubén Orihuela Gavilán es un pionero de la gimnasia rítmica en España. Comenzó a interesarse por este deporte con tan solo 10 años, cuando como el afirma: "me gustaba y no sabía que los chicos no hacían gimnasia” (Orihuela Gavilán R., 2020).

En 1998, y como consecuencia de su petición, sus padres le inscribieron en el Club de Gimnasia Rítmica Sedaví, del municipio Sedaví de la provincia de Valencia. Según dice Rubén "Me dejaron pensando que no duraría mucho" (Ibídem).

Fueron transcurriendo una serie de años, en los que siempre contó con el respaldo de sus padres. Por otro lado, el entorno de sus amigos también le fue favorable. El principal problema que recuerda de sus primeros años fue con el resto de compañeros del colegio. En el recreo se juntaba con las compañeras de gimnasia para jugar y esto no parecía agradar a los demás chicos.

Durante su trayectoria deportiva se proclamó 9 veces campeón de España de gimnasia rítmica en los años 2007, 2008, 2009, 2010, 2011, 2012, 2013, 2014 y 2016. A pesar de haber obtenido todos estos logros deportivos, afirma no haber recibido ningún apoyo por parte de las instituciones competentes. Así, por ejemplo, la RFEG celebraba eventos a los cuales invitaba a las gimnastas que habían conseguido proclamarse campeonas de España cada año y, sin embargo, él, que fue campeón 9 veces, nunca fue invitado.

A pesar de no haber tenido ese reconocimiento institucional, en la actualidad, siente que su valor es reconocido socialmente, ya que muchas personas le apoyan y le han apoyado durante estos años. A nivel personal, con la perspectiva que hoy le permite el tiempo y su posición de entrenador y juez, afirmaba que es consciente de haber alcanzado muchos logros deportivos que le han beneficiado a él y a la gimnasia y que han sido muy gratificantes.

En su testimonio quiso destacar, que se siente muy agradecido con las personas que forman la nueva federación española:

\footnotetext{
"Quiero agradecérselo a ésta; a la de ahora. Al presidente Jesús Carballo, a la nueva seleccionadora individual y excomponente del conjunto nacional subcampeón en los JJOO de Río de Janeiro, Alejandra Quereda, y también, como componente del comité técnico a Isabel Pagán...Estas dos últimas, siempre me han visto ahí a un lado, y ahora están haciendo las cosas diferente... escuchan y sobre todo piden opinión”. (Orihuela Gavilán R., 2020).
} 


\subsection{Los Derechos humanos y el Movimiento Olímpico en la gimnasia rítmica}

En cuanto a los derechos ante la igualdad y el cumplimiento de los Principios Fundamentales de la filosofía olímpica, se podría afirmar que hay un cumplimiento relatico de los mismos dentro de la gimnasia rítmica. A pesar de que es el único deporte, junto con la natación sincronizada, que solo permite la participación femenina en los JJOO, en este momento, esto se debe a que el nivel de representación de esta modalidad aún no cumple las condiciones mínimas para conseguir la participación establecida por el COI.

Podemos afirmar que la práctica de gimnasia rítmica no tiene normativas en cuanto a restricciones de género. Sin embargo, siguen vigentes ciertos prejuicios sociales relacionados con los estereotipos de género, que en España y en Europa se van paulatinamente superando. A pesar de ello, provocaciones e insultos, bromas, ataques e incluso acoso han estado presentes en la trayectoria de Rubén como en la de otros muchos gimnastas. "Para cambiar la percepción social sobre su participación en la gimnasia hay dos vías: visibilidad y educación" afirma Rubén en una entrevista que mantuvo con Hidalgo (2019).

Por otra parte, aunque en estos últimos años se ha aumentado el número de eventos deportivos en los que pueden participar los gimnastas, siguen siendo escasos comparados con los de sus compañeras chicas. Es preciso señalar, que tanto la FIG como las federaciones nacionales tienen en sus manos la posibilidad de generar el impulso que la gimnasia rítmica masculina necesita. Ellas pueden promocionarla, propiciando un aumento de la participación y de la celebración de competiciones, que permitan que ésta pueda llegar a cumplir las cuotas numéricas que incluso le permitan ser olímpica. 


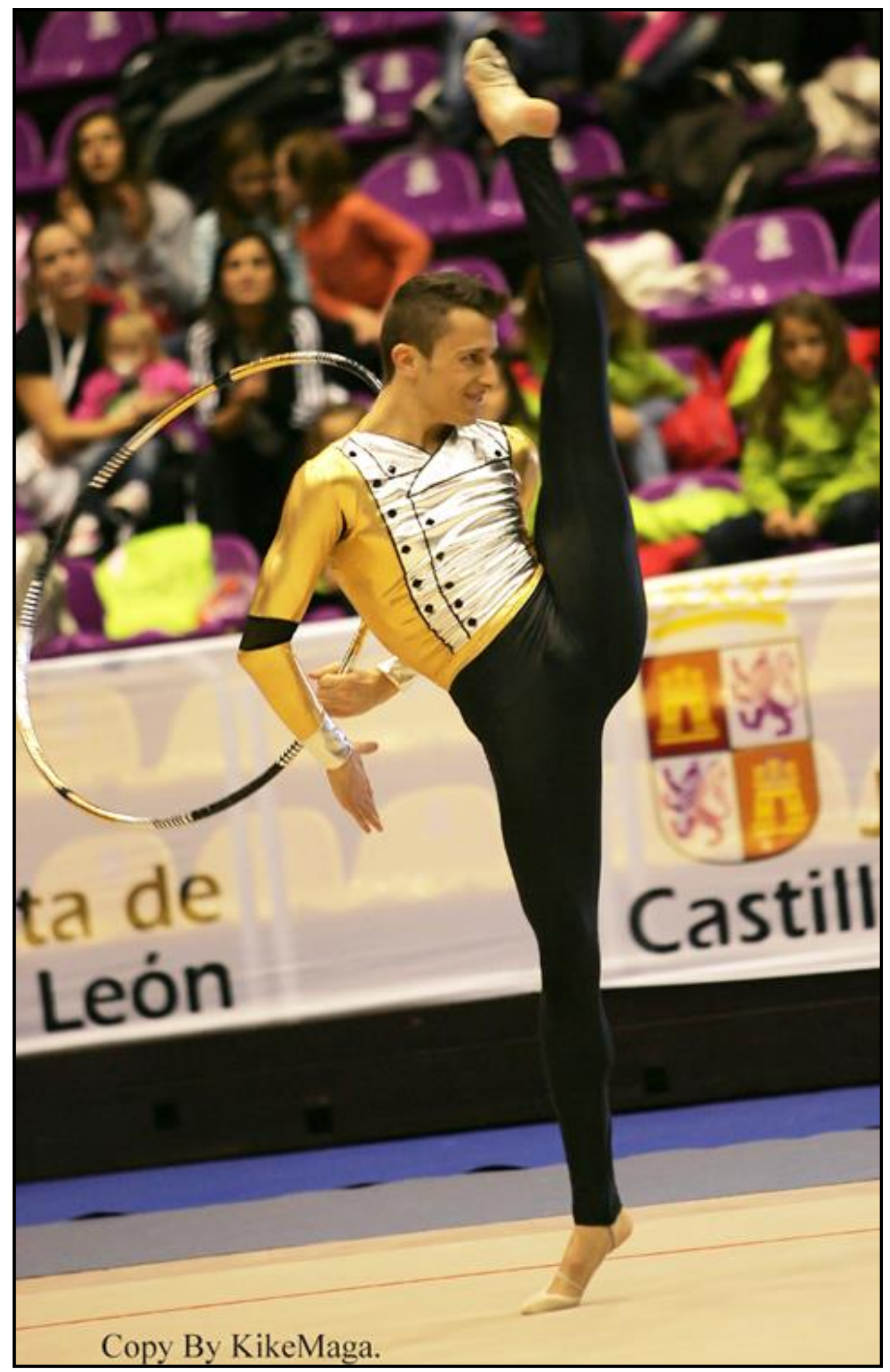

Foto 1. Rubén Orihuela Gavilán durante su participación en el Campeonato de España Masculino en Valladolid 2010. Foto obra de Kike Maga procedente de su archivo personal con su autorización 


\section{Fuentes y Bibliografía}

\subsection{Fuentes Primarias}

\subsubsection{Fuente oral}

- Orihuela Gavilán Rubén. Gimnasta español. Entrevista realizada por videoconferencia en junio de 2020.

\subsubsection{Fuentes de Archivo y escritas}

- Comité Olímpico Internacional (2018) Carta Olímpica. Lausana. Suiza.

- Fotografía de Enrique Magariño. (Valladolid. 2010). Archivo fotográfico personal de Enrique Magariño (Kike Maga).

- ONU: Asamblea General, Declaración Universal de Derechos Humanos, 10 diciembre 1948, 217 A (III), disponible en esta dirección: https://www.refworld.org.es/docid/47a080e32.html

- Real Federación Española de Gimnasia (2020). Gimnasia Rítmica. Normativa. Normativa técnica GR 2020. Recuperado 25 junio de 2020, de: http://rfegimnasia.es/getdata/Getfilenoattachmentmicrosites/name/1676c0cc7b220130d $\underline{\text { af3275b6ec7e0f2.pdf }}$

- Real Federación Española de Gimnasia (2020). Gimnasia Rítmica. Normativa. Listado Provisional $1^{\text {a }}$ Categoría Individual Masculina 2020. Recuperado 25 junio de 2020, de http://rfegimnasia.es/especialidades/normativa/Especialidad/3

\subsubsection{Fuentes hemerográficas}

- Robles, S. (2018). Entrevista a Rubén Orihuela, pionero de la Gimnasia Rítmica Masculina. Recuperado el 8 de junio de 2020, de Sexto Anillo: https://sextoanillo.com/index.php/2018/11/15/ruben-orihuela-pionero-gimnasia-ritmicamasculina/

- Tapigym (2018). Historia y evolución de la gimnasia rítmica masculina II. Recuperado el 8 de junio de 2020, de Tapigym: https://www.tapigym.es/historia-evolucion-gimnasiaritmica-masculina-ii/

- Hidalgo, M. (2019). Así desafían los estereotipos los niños que practican gimnasia rítmica: "Sufren más insultos de lo que la gente imagina". Recuperado el 9 de junio de 2020, de Eldiario.es: https://www.eldiario.es/sociedad/hombres-igualdad-gimnasiaritmica-valientes_0_909559248.html

\subsection{Bibliografía}

- Bédarida, F. (1998). Definición, método y práctica de la Historia del Tiempo Presente. Cuadernos de Historia Contemporánea, 20, 19-27.

- Hammer, D. y Wildavsky, A. (1990). La entrevista semi-estructurada de final abierto. Aproximación a una guía operativa. Historia y Fuente Oral, 4, 23-61. 
- Mendizábal Albizu, S. (2000). Patología en gimnastas de rítmica de alto rendimiento retiradas (Tesis doctoral. Universidad Politécnica de Madrid, España). Recuperado de http://oa.upm.es/1125/1/02200022.pdf

- Soto Gamboa, A. (2004). Historia del presente: estado de la cuestión y conceptualización. HAOL (3) 101-116. Recuperado de: http://dialnet.unirioja.es/descarga/articulo/829443.pdf 